\title{
Implante inmediato de carga inmediata en zona estética: Técnica Socket shield
}

\author{
Immediate implant of immediate load in esthetic zone: Technique Socket Shield
}

\author{
Brenna $\mathrm{ME}^{1}, \mathrm{D}^{\prime}$ itria $\mathrm{JA}^{1}$ \\ Asociación Odontológica de Córdoba
}

\begin{abstract}
Numerous publications have proved the dimensional changes alveolar ridges suffer after a tooth extraction. These changes lead, in most of the cases, to a width's reduction close to 50\%. Trying to minimize this physiological process which occurs after the extraction, different complementary techniques have been implemented in relation to dental implants placing. Many studies have tested how the dental root's permanency in the alveolar bone attains to conserve the tissue dimensions to achieve esthetic restorations. Socket Shield technique, based on these criteria, is applied to avoid structural changes on the buccal bone plate, conserving a dental root portion which remains intact (the vestibular-buccal third), alongside with its periodontium. The dental implant is placed simultaneously to the dental extraction, behind the root remain. The stability and maintenance results of the alveolar bone volume are optimal, being this technique the only one which can achieve that. These results can be translated not only at the gingival margins, but also in the papilla architecture, a very important factor in the fixed prosthesis contours.
\end{abstract}

KEYWORDS: socket, shield, tooth implant, loading, rehability

\section{Resumen}

Numerosas publicaciones han comprobado los cambios dimensionales que sufren los rebordes posteriores a la extracción dentaria. Estos cambios llevan en la mayoría de los casos a una reducción del ancho cercana al 50\%. En el intento de minimizar este proceso fisiológico que se produce luego de la extracción dentaria, se han puesto en práctica distintas técnicas complementarias a la colocación de los implantes dentales. Numerosos estudios han demostrado como la permanencia de restos radiculares en el hueso alveolar consiguen conservar los contornos y volúmenes para el logro de restauraciones estéticas. La técnica "Socket Shield" basada en este fundamento, se realiza con el fin de evitar cambios dimensionales en la pared vestibular del alveolo, conservando intacto una porción de raíz, su tercio corono-vestibular, junto a su periodonto. El implante dental se coloca simultáneamente a la extracción dentaria por detrás del resto. Los resultados de estabilidad y mantenimiento de los volúmenes del proceso alveolar son totales, siendo esta técnica la única que lo logra. Esto se traslada no solo en los contornos gingivales y márgenes, sino también en la arquitectura de la papila, estructura muy importante en los contornos de una rehabilitación fija coronaria sobre implantes.

PALABRAS CLAVE: socket, shield, implante, carga immediata, rehabilitación 


\section{Introducción}

Numerosas publicaciones han comprobado los cambios dimensionales que sufren los rebordes posteriormente a la extracción dentaria. Estos cambios llegan en la mayoría de los casos a una reducción del ancho cercana al 50\% '. Dicha reabsorción resulta, en su mayoría, a expensas de la tabla vestibular, lo que causa una dificultad en la ubicación tridimensional del implante.

En el intento de minimizar este proceso fisiológico que se produce luego de la extracción dentaria, se han puesto en práctica distintas técnicas complementarias a la colocación de los implantes dentales. Entre ellas, la utilización de materiales de injertos es una de las más frecuentes. A pesar de obtener buenos resultados no siempre se logran óptimos contornos en armonía con la rehabilitación protésica. La completa preservación alveolar aún no ha sido documentada en la literatura.

La pronunciada reabsorción ósea es atribuible a la pérdida del ligamento periodontal. Éste es capaz de preservar estructuras óseas de grosor variable, tanto aquellas de gran espesor como corticales sumamente delgadas. El tejido óseo cumple un rol importante en el mantenimiento y estabilidad de la encía.

Numerosos estudios han demostrado cómo la permanencia de restos radiculares en el hueso alveolar consigue conservar los contornos y volúmenes para el logro de restauraciones

estéticas $^{2}$. Björn ${ }^{3}$ demostró la regeneración del hueso alveolar alrededor de restos radiculares, con tratamiento endodóntico, una vez que han sido sumergidos y cubiertos por un colgajo. En 1975, Reames ${ }^{4}$ mostró en animales de experimentación la formación de epitelio y puentes óseos subyacentes sobre restos radiculares incluidos. En 1978 se observó, en preparados histológicos, la formación de nuevo cemento radicular cubriendo totalmente la porción superior del resto, separando la dentina del tejido óseo. Hasta no hace mucho tiempo, se aplicó este método de inclusión ósea de la raíz para preservar el hueso alveolar y lograr soporte en dentaduras completas. También se ha utilizado para conservar la altura de la cresta alveolar en el sitio del fantoma de prótesis parciales a puente". La técnica "Socket Shield" basada en este fundamento, se realiza con el fin de evitar cambios dimensionales en la pared vestibular del alveolo, conservando intacto una porción de raíz, su tercio corono-vestibular, junto a su periodonto (Fig. 1). El implante dental se coloca simultáneamente a la extracción dentaria por detrás del resto.

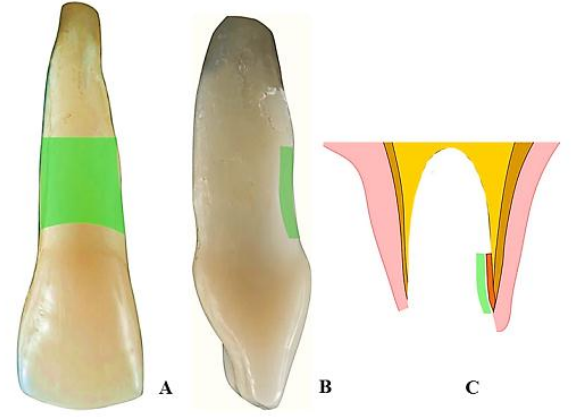

Figura 1. Vista bucal (A) y lateral (B) de la porción de la raíz; Ubicación del resto radicular en el alveolo marcado en verde (C) que actúa como "escudo" marcado en verde

Hürzeler et al. ${ }^{6}$ (2010) y Bäumer et al. ${ }^{7}$ (2015) han reportado los primeros casos con seguimiento clínico de 6 meses a 1.5 años con éxito. Estudios clínicos más recientes con una muestra mayor evidencian el éxito de esta técnica a largo plazo ${ }^{6}$.

\section{Caso clínico nº 1}

Mujer de 50 años, se presenta a la consulta derivada por otro profesional para el reemplazo de un incisivo central superior derecho (elemento 11). En el diagnóstico clínico-radiográfico se observa una fractura radicular. A la inspección con sonda periodontal no presenta alteración alguna (Fig. 2).

El procedimiento se llevó a cabo con anestesia local. La extracción fue realizada sin técnica de colgajo. Se utilizó una piedra diamantada troncocónica para decoronar el elemento dentario. Luego se practicó odontosección preservando intacto el tercio corono-vestibular de la raíz. Una vez que se han dividido ambas partes se procede a la eliminación de la porción radicular a extraer, mediante el uso de elevadores. El segmento radicular más coronal preservado se reduce con piedra de diamante de modo que quede subgingivalmente unos 2 a $3 \mathrm{~mm}$. El alveolo se debridó con curetas pequeñas, cuidadosamente (Fig. 2).

Luego se procede al fresado del alveolo post extracción para la colocación del implante a expensas de la tabla palatina. En primer lugar se utilizó una fresa piloto de diámetro $2,2 \mathrm{~mm}$ logrando la correcta ubicación en las tres dimensiones (ápico-coronario, mesio-distal y vestíbulo-palatino) de manera tal que al continuar con las siguientes fresas de mayor diámetro $(2,8$ $\mathrm{mm}$ y $3,5 \mathrm{~mm}$ ) quede un lecho para la inserción de 
un implante de diámetro $4,1 \mathrm{~mm}$, quedando el mismo por detrás y levemente separado del resto radicular preservado. Se utilizó un implante de nivel óseo, macizo roscado de $4,1 \mathrm{~mm}$ de diámetro y $14 \mathrm{~mm}$ de longitud (Fig. 2), ubicado a $2 \mathrm{~mm}$ de los elementos vecinos, $2 \mathrm{~mm}$ apical del margen gingival, y $1 \mathrm{~mm}$ separado del resto radicular hacia palatino (Fig. 2).

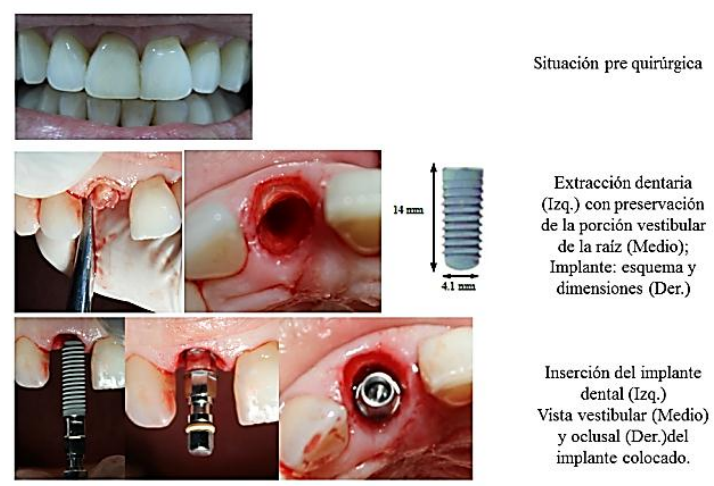

Figura 2. Situación pre-quirúrgica y procedimiento realizado

Inmediatamente después de insertado el implante, se atornilló un pilar para coronas cementadas con una altura de la porción gingival de $1 \mathrm{~mm}$. Se cargó el mismo con una restauración provisional, la que de acuerdo a los volúmenes de emergencia coronaria por vestibular, dejó espacio suficiente y necesario para la proliferación epitelial de la encía libre. La misma selló y cubrió el margen gingival, quedando así el resto radicular sumergido luego de algunas semanas. Se realizan los controles para que en las diferentes trayectorias mandibulares la restauración provisional permanezca fuera de oclusión. Los controles inmediatos y mediatos fueron a las 24 hs, a los 30 días y 60 días. En los mismos se observó la correcta reparación y cicatrización de los tejidos periimplantarios, quedando el resto radicular cubierto por encía (Fig. 3).

Al cuarto mes se corroboró clínicamente la oseointegración del implante. Luego se realizó la toma de impresiones correspondientes, con aditamentos de transferencia para implantes y material siliconado, y se obtuvo un modelo definitivo de trabajo para la restauración definitiva. Se realizó una restauración de porcelana sobre casquillo de zirconio para optimizar los resultados estéticos. La misma se cementó con cementos de resinas compuestas de curado dual. Posterior al cementado de la corona definitiva se realizaron los controles clínicos correspondientes en donde se avala que el elemento protésico definitivo queda absolutamente mimetizado entre los elementos dentarios contiguos y los tejidos periimplantarios. Tanto en su vista frontal como oclusal, no han sufrido ningún tipo de alteraciones dimensionales. Sus papilas, tanto mesial como distal, tampoco han sufrido modificaciones.

Con un estudio radiográfico periapical se confirmó la ausencia de alteraciones en el tejido óseo. Estos altos logros clínicos, estéticos y biológicos persistieron en los controles a los 3 meses, 6 meses, al año y a los 5 años.

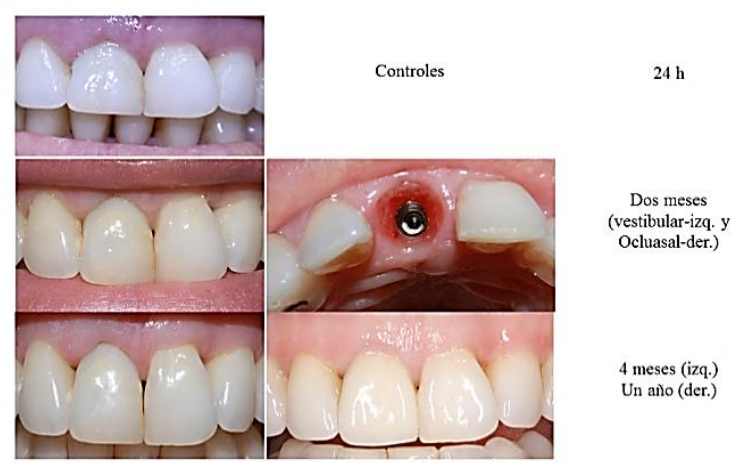

Figura 3. Control a los dos meses (A - Vista vestibular B Vista oclusal), a cuatro meses (C) y a un año (D).

\section{Caso clínico n ${ }^{\circ}$}

Paciente masculino de 33 años, sin antecedentes sistémicos de importancia clínica, se presenta a la consulta con dos restauraciones coronarias decementadas de los elementos 11 y 21 , con percepción personal de gusto y olor desagradable. En el análisis clínico extra e intra oral, el paciente presenta sonrisa alta, biotipo gingival fino, scalloping marcado, dientes de forma coronaria triangular, oclusión estable, sobremordida marcada y con una parafunción de bruxismo. Las dos restauraciones anteriores están soportadas por dos restos radiculares que, a pesar de presentar filtración marginal, se encuentran libres de caries. En el análisis radiográfico de ambos se observa rarefacción en el periápice, con tratamientos de conducto cortas en ambos elementos dentarios (Fig. 4). El paciente decide no someterse a retratamiento de dichas piezas ya que fue sometido a dos tratamientos secuenciales, con un postratamiento doloroso y traumático. Se decide la extracción de los mismos y posterior colocación de implantes. 


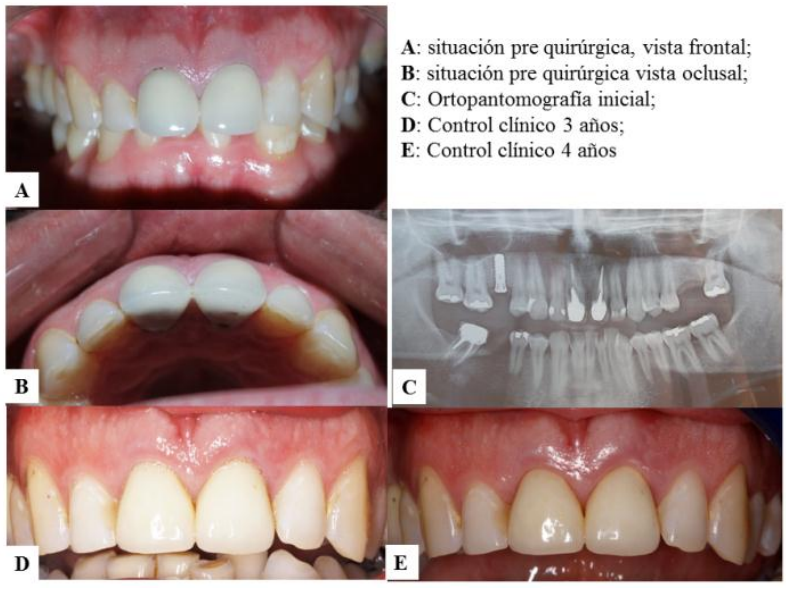

Figura 4. Pre y pos quirúrgico

Se planificó la colocación de implantes en el sitio 11 y 21, en conjunto con la implementación de la técnica "socket shield" (Fig. 5). En este caso se utilizaron implantes de nivel tisular, que incluyen transmucoso de $1,8 \mathrm{~mm}$ de altura.

Los controles se realizaron con el mismo protocolo anteriormente descripto, en donde la evolución es absolutamente favorable.

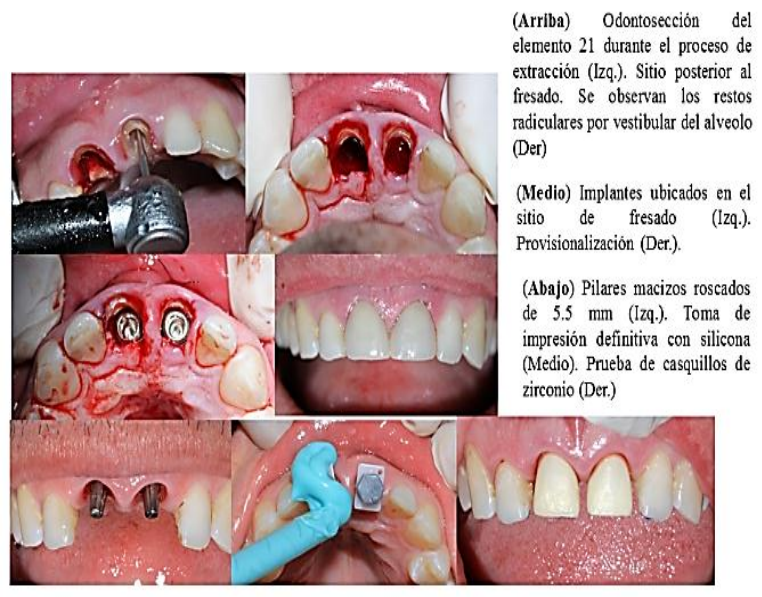

Figura 5. Etapas del procedimiento realizado

Los logros estéticos en este caso son aún más marcados debido a que las papilas, sobre todo las inter-implantarias son agudas y largas, en donde la mínima pérdida dimensional de la misma tendría una alta repercusión en el festoneado gingival. Desde el punto de vista dimensional no se observaron modificaciones algunas en todos sus planos. Lo más destacado fue la estabilidad de los tejidos periimplantarios a lo largo de los 3 meses, 6 meses, un año y 3 años siguientes.

\section{Discusión}

Una vez más, a través de estos dos casos, como así también con los antecedentes de estudios experimentales como clínicos, la preservación de la porción vestibular más coronaria del resto radicular simultánea a la colocación del implante, no solo se manifiesta con una ausencia total de un proceso inflamatorio crónico y de reabsorción ósea. Por lo contrario, los resultados de estabilidad y mantenimiento de los volúmenes del proceso alveolar son totales, siendo esta técnica la única que lo logra. Esto se traslada no solo en los contornos gingivales y márgenes, sino también en la arquitectura de la papila, estructura muy importante en los márgenes de una rehabilitación fija coronaria sobre implantes. Esto último toma mucha importancia en el segundo caso clínico, en donde se sabe que la colocación de dos implantes contiguos, da como resultado la pérdida de la cresta ósea entre los mismos dando como consecuencia una marcada reducción de las papilas, y en algunos casos, su total desaparición. Para entender que este fenómeno fisiológico reabsortivo después de la extracción se produce debido a la pérdida del hueso fibro-dependiente (bundle bone) y del ligamento periodontal, y para conservar el contorno gingival, se deben preservar estas estructuras que le dan el aspecto natural a los tejidos

Todos los autores declaran que no existen conflictos potenciales de interés con respecto a la autoría y / o publicación de este artículo.

All authors declare no potential conflicts of interest with respect to the authorship and/or publication of this article.

\section{Referencias}

1. Araújo MG \& Lindhe J. Dimensional ridge alterations following tooth extraction. An experimental study in the dog. Journal of Clinical Periodontology 2005; 32, 212 218.

2. Andersson L, Emami-Kristiansen Z, Hogstrom J. Singletooth implant treatment in the anterior region of the maxilla for treatment of tooth loss after trauma: a retrospective clinical and interview study. Dent Traumatol. 2003; 19(3):126-31.

3. Björn H. Free transplantation of gingival propria. Sven Tandlak Tidskr 1963; 22: 684.

4. Reames RL, Nickel J S, Patterson SS, Boone M, elKafrawy AH. Clinical, radio-graphic, and histological study of endodontically treated retained roots to preserve alveolar bone. Journal of Endodontics. 1975; 1, 367373. 
5. Salama M, Ishikawa I, Salama H, Funato A, Garber DA. Advantages of the Root Submergence technique for Pontic Site Develop- ment in esthetic implant therapy. International Journal of Periodontics \& Restorative Dentistry. 2007; 27, 521-527.

6. Hürzeler MB, Zuhr O, Schupbach $\mathrm{P}$, Rebele SF, Emmanouilidis N, Fickl S. The socket-shield technique: a proof-of-principle report. J Clin Periodontol. 2010; 37(9):855-62.
7. Bäumer D, Zuhr O, Rebele S, Hürzeler M. Socket Shield Technique for immediate implant placement clinical, radiographic and volumetric data after 5 years. Clin Oral Implants Res. 2017; 28(11):1450-1458.

Correspondencia a/ Corresponding to: Dr. José A Dítria Asociación Odontológica de Córdoba San Jerónimo 177, $2^{\circ}$ piso, X5000 Córdoba Argentina 